\title{
Machine Learning Facial Emotion Recognition in Psychotherapy Research. A useful approach?
}

\author{
Martin Steppan ${ }^{\star 1}$, Ronan Zimmermann ${ }^{1,2}$, Lukas Fürer ${ }^{1}$, Nathalie Schenk ${ }^{1}$, and \\ Klaus Schmeck ${ }^{1}$ \\ ${ }^{1}$ Child and Adolescent Research Department, Psychiatric University Hospital, \\ Basel, University of Basel \\ ${ }^{2}$ Division of Clinical Psychology, Faculty of Psychology, University of Basel
}

\begin{abstract}
Background: Manual coding of facial emotion expression, e.g. using the Facial Action Coding System (FACS), can be very time consuming. For psychotherapy research the change in emotion expression and microexpressions over time is relevant. Hence, automatic emotion recognition may be a promising tool. Method: We apply a Convolutionary Neural Network (CNN) for emotion recognition to video material from 389 psychotherapy sessions of 23 patients with borderline personality pathology. We cross-validate the findings with human ratings according to the Clients Emotional Arousal Scale (CEAS) and the outcome of psychotherapy. Results: Overall, machine learning ratings show substantial, however, numerically low agreement with human ratings, particularly with overall (non-specific) emotional arousal. Machine learning emotion recognition shows substantial predictive validity for therapy outcome, in particular the display of positive emotions (smiling and happiness). Discussion: Machine learning is a highly promising resource for tracking change in emotional expression over time. The results highlight the differential association of displayed positive and negative feelings to the treatment outcome.
\end{abstract}

Keywords: Emotion recognition - Video · Affective Computing · Psychotherapy research $\cdot$ Psychotherapy process research

\footnotetext{
* corresponding author: Dr Martin Steppan, Universitäre Psychiatrische Kliniken, Wilhelm Klein Strasse 27, 4002 Basel, Switzerland; Email: mhsteppan@gmail.com
} 


\section{Introduction}

Facial emotional expression has been intensively studied in psychology, most famously represented by Ekman's Facial Action Coding System (FACS, Ekman, 1997). Since FACS-codings are highly time-consuming, the applications within psychotherapy often had to be carried out using single case studies (BänningerHuber, 1992). Since automatic emotion recognition software has become available only in recent years (e.g. Facereader), only one first study has already pioneered the field applying machine learning emotion recognition (MLER) to the field of psychotherapy (Arango et al., 2019). This lack of studies is in stark contrast to a wide range of studies on verbal emotion expression in psychotherapy (Peluso Freund, 2018). Hence, as opposed to other forms of evaluating emotional expression, the reliability and validity of these machine learning approaches is yet widely unknown. The aim of this study is to test whether MLER is a reliable and valid tool for psychotherapy research.

\section{Methods}

\subsection{Ethical approval}

This study is part of the multi-centre study 'Evaluation of Adolescent Identity Treatment' (Schmeck et al., 2018; Zimmermann et al., 2018) that has been registered at clinicaltrials.gov (NCT02518906). The current analyses are based on the entire available data collected at one participating centre (Psychiatric University Hospitals, Basel). Ethical approval was obtained from the local ethics committee. All adolescents, their parents and the therapists provided written informed consent for participation.

\subsection{Sample}

The sample consists of 23 adolescent patients with borderline personality pathology (Structured Clinical Interview for DSM-IV Axis II Personality Disorders: SCID-II; First, Gibbon, Spitzer, Williams, Benjamin, 1997) and identity diffusion according to the Assessment of Identity Development in Adolescence (AIDA) (total t score >60) (Goth et al., 2012; Lind, Vanwoerden, Penner, Sharp, 2019). The mean age of the patients was 16.2 years $(\mathrm{SD}=1.6$ years) years. The original material consisted of footage of 423 therapy sessions (duration approx. 50 minutes). Thirty-two videos were excluded mainly due to errors in face detection, i.e. the algorithm constantly detected a background object as a face.

\subsection{Video processing and machine learning}

Figure 1 shows the setting of psychotherapy sessions in our lab in Basel. Open source Python scripts have been adapted and used to process videos. For face detection OpenCV was used (Bradski, 2000). Since faces are relatively stationary 
throughout a therapy session, face detection was optimized by dynamically defining an area of high likelihood of detecting a face. Only if no face was detected for more than one second, the region of interest was extended. In doing so, a high accuracy of face detection could be achieved. To test this empirically, every 100 frames, a picture of the selected region of interest was saved to quantify the accuracy of face detection. For emotion detection, an open source model which is based on a Convolutional Neural Network $(\mathrm{CNN})$ has been implemented. This network had been trained using the "FER-2013 dataset", a collection of 35,685 face pictures designed to develop machine learning algorithms for facial emotion recognition (Goodfellow et al., 2013). Similar to previously used algorithms, e.g. the software FaceReader (Arango et al., 2019), probabilities of the occurrence of six basic emotions (happiness, surprise, anger, disgust, sadness, fear), plus a percentage of neutral expression were extracted. Altogether, including also the videos of therapists (data not used here), we analyzed more than one month of video material. Hence, in order to reduce calculation time, calculations were performed at sciCORE (http://scicore.unibas.ch/) - a high performance cluster computer and scientific computing center - at University of Basel. The full python script which has been used, can be found here.

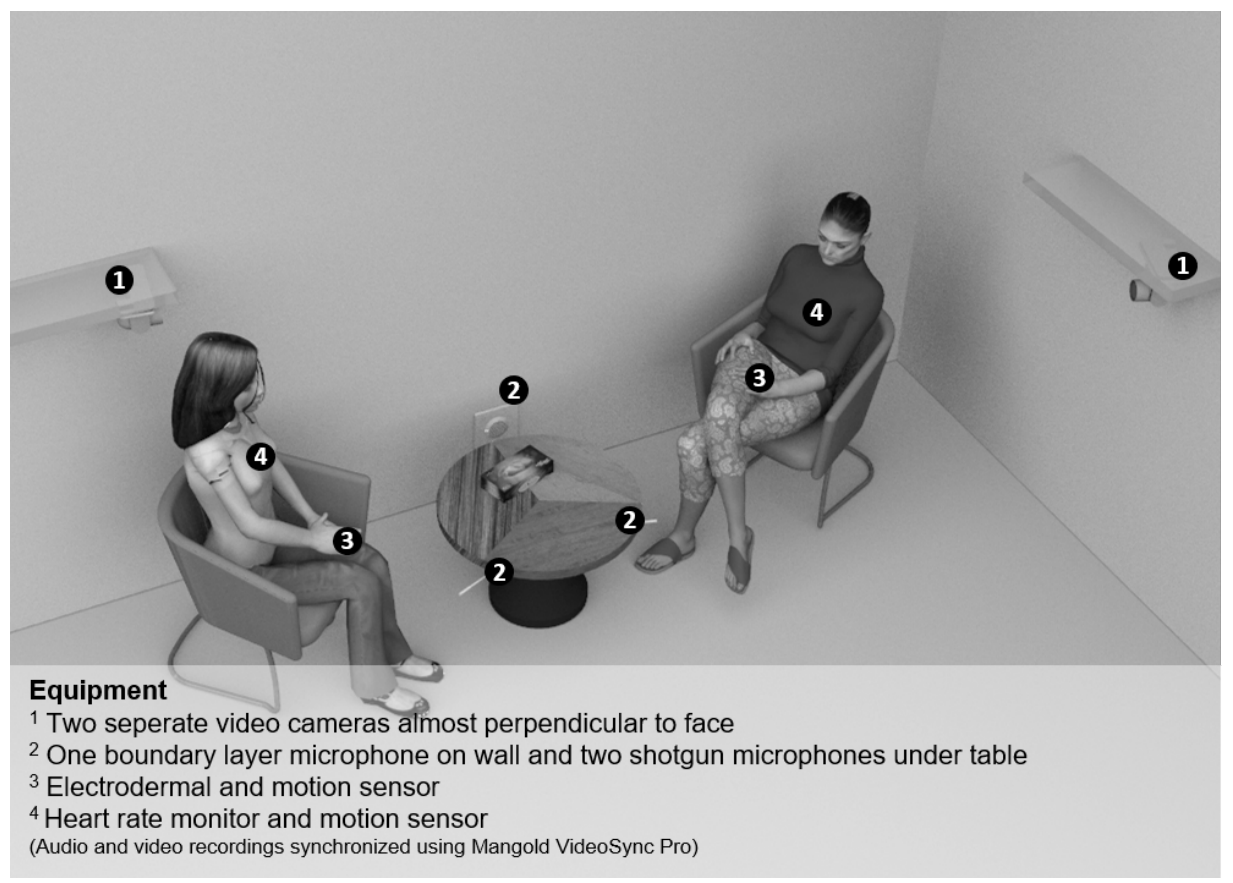

Fig. 1: Setting and technical infrastructure of psychotherapy sessions (symbolic visualization) 


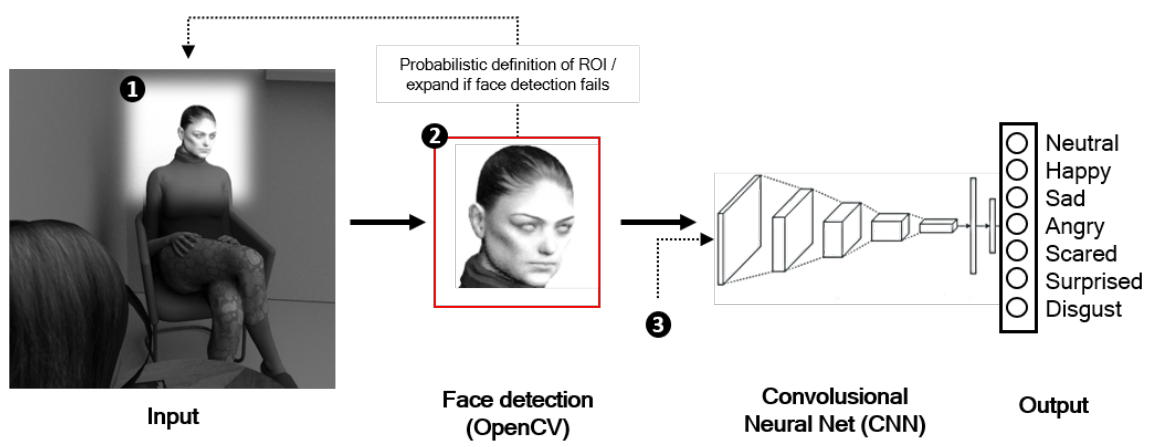

Fig. 2: Architecure of image processing. $1=$ to increase speed and reduce error, a region of interest (ROI) can be defined where a face is detected with high likelihood which is expanded if face detection fails; $2=$ Faces are detected and transformed into 48x48 pixels grayscale pictures; $\mathrm{x}$ and $\mathrm{y}$ coordinates width and height of deteced face are recorded for error detection and cross-validation of motion-sensors; $3=$ The convolusional neural net had been trained using the "FER-2013 dataset".

\subsection{Client Emotional Arousal Scale}

Three observers were trained to use the client emotional arousal scale (CEAS). For each minute interval the CEAS provides a rating between 1 and 7 (ordinal Likert scale, $1=$ low $/$ no arousal, $7=$ full arousal) measuring the intensity of the patient's arousal and a rating of the predominant emotional valence (joy, sadness, fear, anger, disgust, love). For intervals which were rated low in emotional arousal $(<3)$, no specific emotion was rated. Raters were involved in a training phase, involving rating 7 sessions followed by a consensus rating. A total of 232 sessions were rated (on average 9.8 sessions per dyad). Altogether 11,960 minute intervals (i.e. 186.5 hours) of video material were rated manually. Inter-rater reliability has been reported from 0.75 to 0.81 in previous studies (Greenberg et al. 2007, Missirlian et al., 2005).

\subsection{External criteria for reliability and validity}

In order to quantify reliability, i.e. the agreement of MLER with human observers, we cross-validated the findings using the Clients Emotional Arousal Scale (CEAS; Machado, 1992; Rosner, 1996). To test clinical validity, i.e. the importance of MLER for therapeutic outcome, we cross-validated the findings with pre- and post-measures on several psychological questionnaires. These included subscales of the Youth Outcome Questionnaire (Y-OQ; Burlingame et al., 2004): Intrapersonal Distress, Somatic, Interpersonal Relationships, Social Problems, Behavioural Dysfunction; subscales of the Level of Personality Functioning Questionnaire (LoPF; 
Goth, Birkholzer and Schmeck, 2018): Self Direction, Empathy, Identity and Intimacy; as well as the Zanarini Rating Scale for Borderline Personality Disorder (ZAN-BPD, Zanarini, 2007). Six individuals dropped out from psychotherapy, so that therapy outcome was available for 17 individuals.

\subsection{Statistical analysis}

We aimed at using raw data wherever possible, i.e. we did not intend to perform highly complex adaptations to the data in order to improve reliability or validity, to show the unadorned strengths and weaknesses of MLER. For unspecified emotional arousal according to human raters (CEAS-R), we calculated Pearson's correlation of CEAS-ratings with the display of a neutral facial expression according to MLER. We report this correlation also after standardizing within each individual (i.e. equivalent to a random intercept model), and individually for three different human raters. Using an example therapy, we showcase the temporal agreement of unspecific CEAS-arousal ratings and MLER neutral facial expression over the course of psychotherapy. This individual result is compared to the overall association in all individuals and all sessions. For more specific human ratings using CEAS, we calculated Pearson correlations of all seven MLER scores with the specific emotions labelled by human raters. Specific emotions were only labelled by human raters when CEAS-ratings were higher than 2 .

Regarding continuous therapy outcome we calculated a simple sum score using all aforementioned outcome subscales before and after treatment, and calculated the difference as a simple metric of change over time (delta). Using mediansplitting we allocated individuals to two groups, i.e. good outcome and poor outcome. Two individuals showed a higher symptom count after treatment than before. In order to show the difference between these two groups we calculated independent sample t-tests for all seven MLER categories, and calculated Cohen's $\mathrm{d}$ as a measure of effect size. Regarding continuous therapy outcome, we calculated Pearson correlations between pre- and post symptom score, delta and each MLER category. We tested the robustness of these correlations by a stepwise removal of individuals which contribute most to the significance of the correlations.

For all of these analyses, the unit of analysis was minutes, i.e. we used MLER scores averaged over minutes. This is in correspondence with human CEAS-rating which were also done for minute intervals of the video material. All analyses and visualization were done in R Studio (RStudio Team, 2015) using version 3.5.3 of the $\mathrm{R}$ programming language. For visualization purposes we used the package ggplot2 (Wickham, 2016). 


\section{Results}

\subsection{Agreement with human raters - overall arousal}

Figure 3 illustrates the agreement of human raters and machine learning emotion recognition. Video sequences, which were rated as highly emotional by humans (CEAS > 3), were also classified as less neutral by MLER. The effect is stable when controlling for individual differences in overall display of a neutral facial expression (i.e. a standardization of $\mathrm{y}$-values within each individual: $\mathrm{r}=-.070$; $\mathrm{p}<1.39 \mathrm{e}-11)$. Although the level of significance is very stable, the underlying correlation is small in size $(\mathrm{r}=-.104, \mathrm{p}<6.46 \mathrm{e}-24)$. However, for three human raters, the agreement of CEAS-arousal ratings with machine learning neutral ratings was highly comparable given three similar correlations of $\mathrm{r}=-.11, \mathrm{r}=-.10$ and $\mathrm{r}=-.10$ respectively (for each rater $\mathrm{p}<10 \mathrm{e}-10$ ). Figure 4 shows the agreement of MLER and human ratings for eight psychotherapeutic sessions of one example individual in the sample. The results are compared to the overall effect for all individuals and all sessions (see histogram in Figure 4).

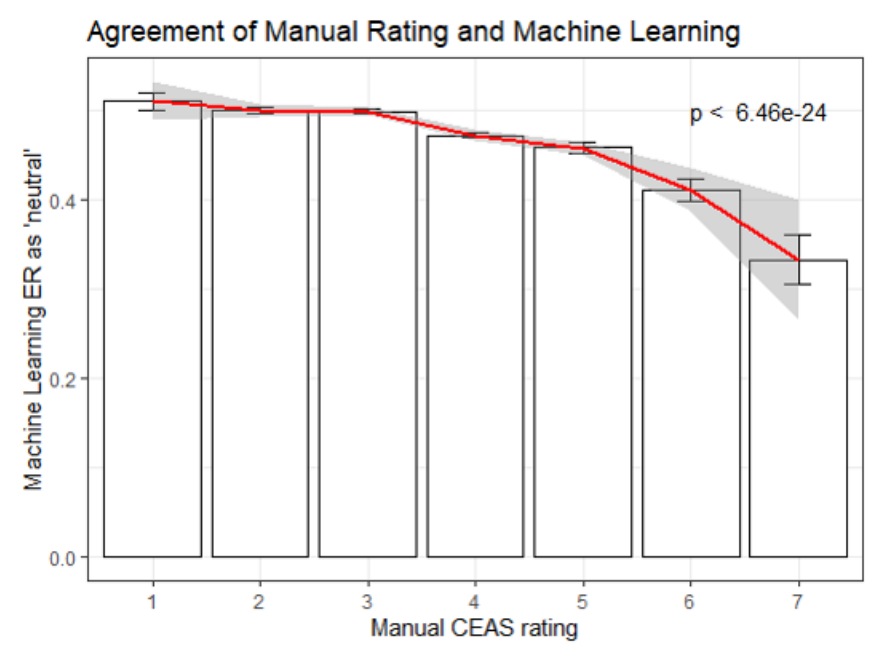

Fig. 3: Agreement between human raters and machine learning algorithm $(\mathrm{N}=9,298$ rated minutes; on average 1,055 valid video frames per rated minute, i.e. $9,813.981$ frames) 
Human CEAS-Rating - MLER
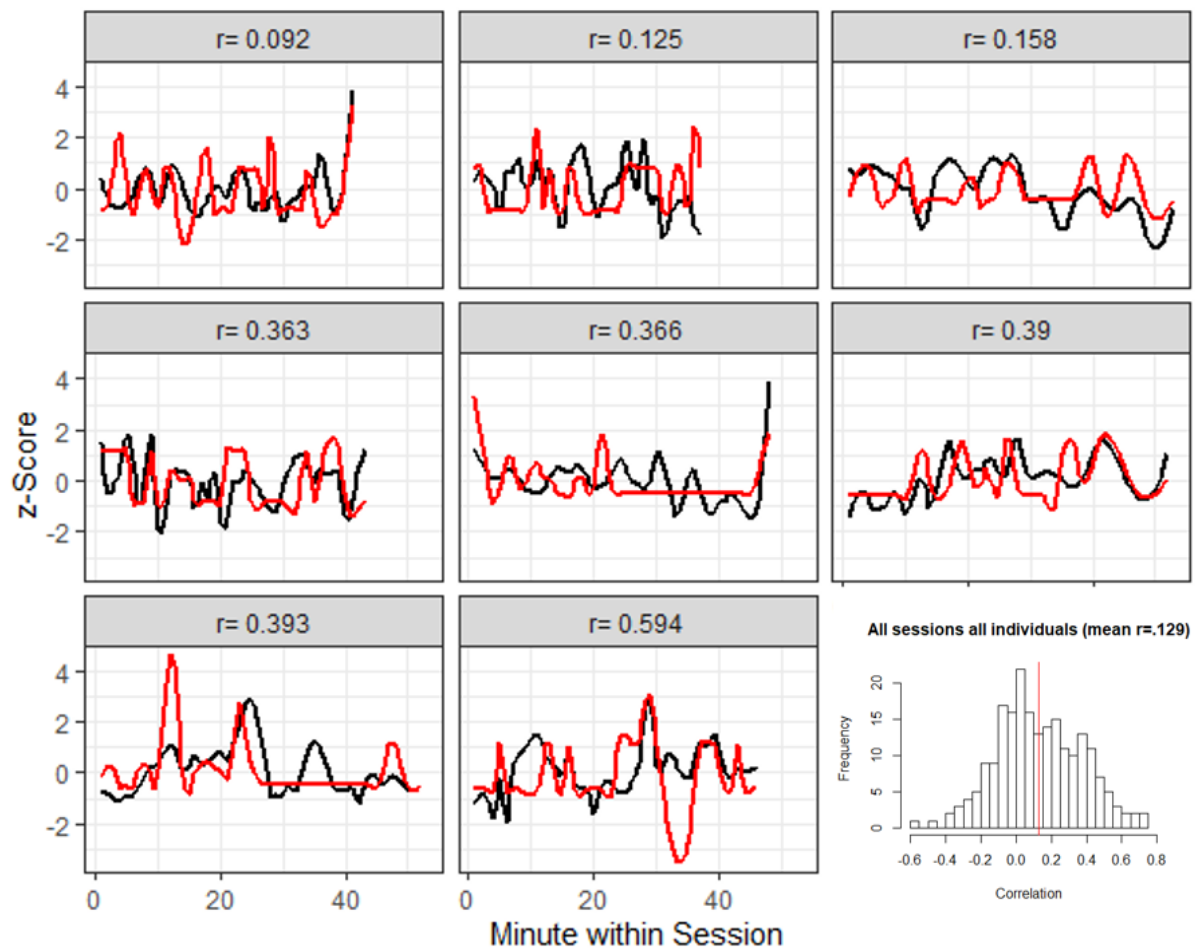

Fig. 4: Agreement of manual CEAS-rating and MLER in eight psychotherpeutic sessions in one example individual. Histogram shows the equivalent correlations for all individuals and all sessions (red line indicates average correlation). Note: the y-axis represents MLER inverse value for neutral facial expression (i.e. 1 neutral). Both MLER and human rating are standardized) 


\subsection{Agreement with human raters - specific emotions}

Figure 5 shows correlations between specific emotions rated by human raters and machine learning emotion recognition. For all specific emotions, except for fear (scared), correlations are positive and significant (see diagonal correlations). The correlation matrix is not very specific for each emotion: except for the expected significant correlations along the diagonal, there are several other significant associations, e.g. angry and scared $(\mathrm{r}=.02)$, or surprised and sad $(\mathrm{r}=.13)$. Hence, MLER scores are only partly useful to classify which emotion had been rated by humans (only anger exceeds a 50 percent chance to be correctly classified using multinomial logistic regression).

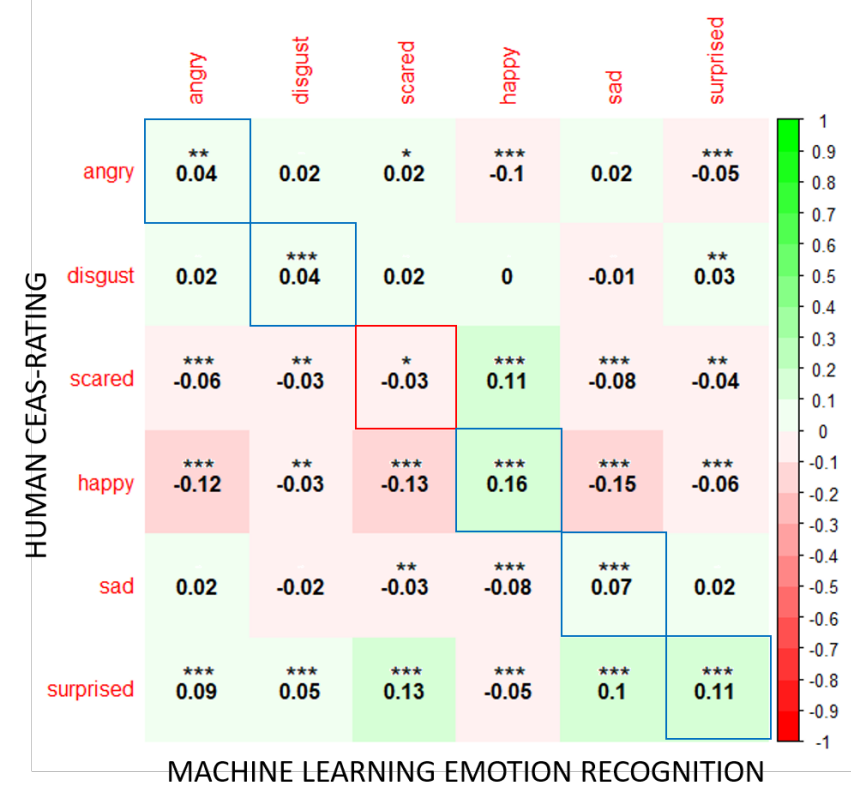

Fig. 5: Correlation matrix - agreement between human raters and machine learning algorithm for specific emotions with CEAS rating $>2(\mathrm{~N}=5,867$ rated minutes; on average 1,020 valid video frames per rated minute, i.e. 5,988.254 frames) 


\subsection{Clinical Validity}

\subsection{Dichotomized therapy outcome}

Figure 6 illustrates the association of therapy outcome and the display of emotions within the therapeutic process (all sessions, as measured by MLER). Patients with a poorer outcome $(<$ Median) displayed significantly more anger, more disgust, less happiness, more neutral facial expressions, more sadness, more fear and more surprise. The most pronounced effect was found for happiness with an effect size of Cohen's $d=0.57(\mathrm{p}<.43 \mathrm{e}-254)$, indicating that patients with successful therapies displayed significantly more happiness than patients with less successful therapies.

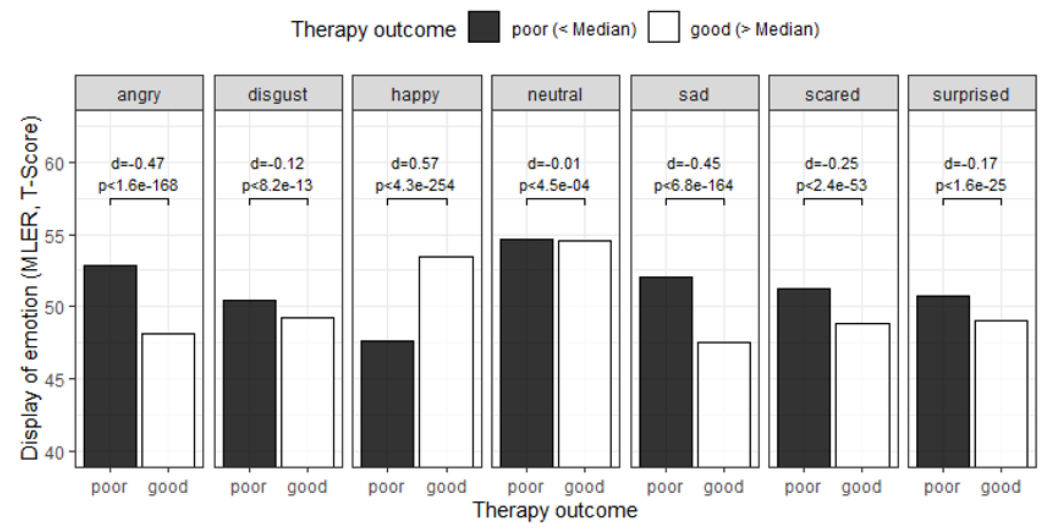

Fig. 6: Display of emotions based on MLER and dichotomized therapy outcome (18,064.823 analyzed frames collapsed into $\mathrm{N}=18,658$ minute intervals (mean emotion per minute); on average 1,009 valid video frames per minute) 


\subsection{Continuous symptomatology before and after treatment}

Table 1 shows correlations of MLER and pre- and post Borderline personality symptomatology, as well as the improvement between pre- and post-assessment (Delta). While display of specific emotions is only weakly associated with the symptomatology at the beginning of the therapy, higher correlations were observed with the post-symptomatology at the end of the treatment. In particular, happiness (negative), sadness (positive), anger (positive) and neutral facial expressions (positive), were correlated with more symptoms at the end of the treatment. Hence, the improvement over time (Delta), was associated with the same emotions in the opposite direction. These findings suggest that it is not the baseline symptomatology, but rather the degree of improvement which is associated with display of emotions throughout therapy.

\begin{tabular}{lcrcrcr}
\hline & Pre & Post & Delta & $\mathrm{p} 1$ & $\mathrm{p} 2$ & $\mathrm{p} 3$ \\
\hline Emotion & Pearson & Pearson & Pearson & $\mathrm{p}<$ & $\mathrm{p}<$ & $\mathrm{p}<$ \\
\hline angry & -0.02 & 0.19 & -0.18 & $1.8 \mathrm{e}-02$ & $4.8 \mathrm{e}-129$ & $5.5 \mathrm{e}-119$ \\
disgust & -0.13 & -0.04 & -0.02 & $1.5 \mathrm{e}-66$ & $9.5 \mathrm{e}-08$ & $2.3 \mathrm{e}-03$ \\
scared & 0.12 & 0.10 & -0.05 & $3.0 \mathrm{e}-57$ & $1.6 \mathrm{e}-35$ & $3.1 \mathrm{e}-11$ \\
happy & -0.01 & $-0.37^{*}$ & $0.38^{*}$ & $8.9 \mathrm{e}-02$ & $0.0 \mathrm{e}+00$ & $0.0 \mathrm{e}+00$ \\
sad & 0.00 & $0.22^{*}$ & $-0.25^{*}$ & $7.2 \mathrm{e}-01$ & $2.2 \mathrm{e}-162$ & $1.2 \mathrm{e}-218$ \\
surprised & 0.08 & 0.07 & -0.05 & $1.1 \mathrm{e}-25$ & $3.5 \mathrm{e}-20$ & $4.3 \mathrm{e}-10$ \\
neutral & -0.03 & 0.15 & -0.17 & $6.8 \mathrm{e}-05$ & $3.9 \mathrm{e}-81$ & $1.1 \mathrm{e}-104$ \\
\hline
\end{tabular}

Table 1: Correlation matrix. Pre $=$ Symptom count before treatment, Post $=$ symptom count after treatment, Delta $=$ difference between pre and post indicative of positive development; ${ }^{*}$ correlations are highlighted which are most robust, i.e. remain significant $(\mathrm{p}<.05)$ when excluding three individuals which contribute most to the correlation 


\section{Discussion}

In this pilot study we tested the most basic quality criteria of a scientific tool, i.e. reliability and validity. For both criteria, we found substantial evidence, indicating that machine learning emotion recognition (MLER) is most likely a valuable tool for psychotherapy research. MLER shows substantial, however numerically low, agreement with human CEAS-ratings. It could also be shown that emotions displayed throughout therapy, as assessed through MLER, are strongly associated with therapy outcome. Based on these basic findings, we emphasize the importance to further investigate MLER as a tool for psychotherapy research.

Regarding reliability (agreement with human raters) the results are highly significant, numerically low and not very specific. This may have several reasons: a) human raters in this study were not advised to rate facial expressions, but to evaluate the emotional valence of the situation, including also verbal and nonverbal communication. b) human raters could only select a primary emotion for each rated minute, whereas MLER produces percentage scores for each emotion for each video frame. This mismatch may be one reason why the associations are not very specific. c) The unit of analysis (1-minute intervals) is relatively long to detect specific emotions. It can be assumed that over the course of 1 minute several different emotions can emerge). Nonetheless, the results clearly support reliability, and more so objectivity, of MLER, supporting the evidence of earlier findings by Arango et al. (2019). The results indicate high objectivity of MLER, as the agreement of three human raters with MLER are almost identical.

Regarding therapy outcome we found striking results. These results are in line with earlier findings applying the Facial Action Coding System (FACS), highlighting the importance of smiling and positive feelings in psychotherapy (Bänninger-Huber, 1992). The fundamental statistical problem of this analysis is that data is nested within 17 individuals for which therapy outcome is available. However, we only found robust associations with post-treatment symptomatology and change over time, but not with pre-treatment symptomatology. Hence, the results suggest that the display of positive emotions is more a function of the treatment and not of the pre-existing personality disposition of the individuals. This may be promising for more complex, e.g. auto-regressive longitudinal statistical methods.

Several limitations of this approach are worth considering: 1) The applied machine learning algorithms are a "patchwork" of open source algorithms. More specialized software products, e.g. in robotics, may exist, but are not publicly available. Hence, the applied MLER may underestimate the true potential of this methodology. 2) The applied machine learning algorithms are not tailored to the individual. An even better outcome can be expected if the algorithms were trained to each individual. However, we did not use this approach to avoid over-fitting and increase the generalizability of the results. 3) The sample we used is relatively specific (adolescents with Borderline personality pathology). Hence, results may be different in other samples. The comparison of emotional expression as measured by MLER and its association to outcome in different patient groups and treatments appears to be a formidable direction for future research. 4) 
Unfortunately, the MLER approach used in this study must be considered a computationally heavy weight. Extraction of the data would not have been possible in such a short time without the mentioned scientific computing center.

This pilot study is clearly supporting the approach to use machine learning emotion recognition for psychotherapy. Far away from being a "perfect tool", the method still shows a high potential for predicting therapy outcome. Given more qualitative, and not quantitative attempts prior to this study, the method is also highly valuable in "data-mining" emotionally relevant video sequences (e.g. alliance ruptures). The high temporal resolution of MLER (about 25-30 frames per second) makes the approach highly promising for analyzing microexpressions. The combination and complementation of MLER with automatized emotion recognition from the voice will yield a richer picture and possibly provide feasible methodology for extracting longitudinal emotional arousal over the course of therapies. Given that we also applied MLER to footage of our therapists, we will be able to provide information on emotional synchronicity, emotional dominance / leading and related metrics in the future. Nonetheless, this study also shows that a larger sample size would be important. Hence, we encourage the constitution of a consortium with other research centers on this topic, in order to share anonymized meta-data for this purpose. 


\section{References}

1. Arango, I., Miranda, E., Ferrer, J. C. S., Fresán, A., Ortega, M. A. R., Vargas, A. N., ... Robles, R. (2019). Changes in facial emotion expression during a psychotherapeutic intervention for patients with borderline personality disorder. Journal of psychiatric research, 114, 126-132.

2. Bänninger-Huber, E. (1992). Prototypical affective microsequences in psychotherapeutic interaction. Psychotherapy Research, 2(4), 291-306.

3. Bradski, G. (2000). The OpenCV Library. Dr. Dobb39;s Journal of Software Tools.

4. Ekman, R. (1997). What the face reveals: Basic and applied studies of spontaneous expression using the Facial Action Coding System (FACS). Oxford University Press, USA.

5. Eubanks, C. F., Burckell, L. A., Goldfried, M. R. (2018). Clinical consensus strategies to repair ruptures in the therapeutic alliance. Journal of psychotherapy integration, $28(1), 60$.

6. First, M. B., Gibbon, M., Spitzer, R. L., Williams, J. B. W., Benjamin, L. S. (1997). Structured Clinical Interview for DSM-IV Axis II Personality Disorders (SCID-II). Washington, DC: American Psychiatric Press.

7. Goth, K., Birkhölzer, M., Schmeck, K. (2018). Assessment of Personality Functioning in Adolescents With the LoPF-Q 12-18 Self-Report Questionnaire. Journal of personality assessment, 100(6), 680-690.

8. Goodfellow, I. J., Erhan, D., Carrier, P. L., Courville, A., Mirza, M., Hamner, B., ... Zhou, Y. (2013, November). Challenges in representation learning: A report on three machine learning contests. In International Conference on Neural Information Processing (pp. 117-124). Springer, Berlin, Heidelberg.

9. Goth, K., Foelsch, P., Schlüter-Müller, S., Birkhölzer, M., Jung, E., Pick, O., Schmeck, K. (2012). Assessment of identity development and identity diffusion in adolescence-Theoretical basis and psychometric properties of the self-report questionnaire AIDA. Child and Adolescent Psychiatry and Mental Health, 6(1), 27. https://doi.org/10.1186/1753-2000-6-27

10. Lind, M., Vanwoerden, S., Penner, F., Sharp, C. (2019). Inpatient adolescents with borderline personality disorder features: Identity diffusion and narrative incoherence. Personality Disorders: Theory, Research, and Treatment, 10(4), 389-393. https://doi.org/10.1037/per0000338

11. Machado, P.P.P. (1992). Client's emotional arousal in therapy: Development of a rating scale. Unpub- lished manuscript, Psychotherapy Research Project, University of California, Santa Barbara.

12. Peluso, P. R., Freund, R. R. (2018). Therapist and client emotional expression and psychotherapy outcomes: A meta-analysis. Psychotherapy, 55(4), 461.

13. RStudio Team (2015). RStudio: Integrated Development for R. RStudio, Inc., Boston, MA URL http://www.rstudio.com/.

14. Rosner, R. (1996). The relationship between emotional expression, treatment, and outcome in psychotherapy. Frankfurt: Lang.

15. Ramseyer, F. T. (2019). Motion Energy Analysis (MEA). A primer on the assessment of motion from video. Journal of Counseling Psychology.

16. Schmeck, K., Pick, O. G., Milidou, M., Schenk, N., Schlüter-Müller, S., Zimmermann, R. (2018). Früherkennung von Persönlichkeitsstörungen. PTTPersönlichkeitsstörungen: Theorie und Therapie, 22(3), 179-185.

17. Stiles, W. B. (2002). Session evaluation questionnaire: Structure and use. Journal of Clinical Psychology, 55, 10-12. 
18. Wells, M. G., Burlingame, G. M., Lambert, M. J. (1999). Youth Outcome Questionnaire (Y-OQ).

19. Wickham, H. (2016). ggplot2: Elegant Graphics for Data Analysis. Springer-Verlag New York. ISBN 978-3-319-24277-4, https://ggplot2.tidyverse.org.

20. Zanarini, M. C. (2007). Zanarini Rating Scale for Borderline Personality Disorder (zan-bpd). Jones Bartlett Learning.

21. Zimmermann, R., Fürer, L., Schenk, N., Koenig, J., Roth, V., Schlüter-Müller, S., Kaess, M., amp; Schmeck, K. (under review). Silence in the Psychotherapy of Adolescents with Borderline Personality Pathology-Associations with Personality Pathology and Session Impact. Personality Disorders: Theory, Research, and Treatment. 